\title{
Upregulation of Mitochondrial Transcription Factor A Promotes the Repairment of Renal Tubular Epithelial Cells in Sepsis by Inhibiting Reactive Oxygen Species-Mediated Toll-Like Receptor 4/p38MAPK Signaling
}

\author{
Xin-Gui Dai ${ }^{\mathrm{a}}$ Tao Li $^{\mathrm{b}}$ Wei-Bo Huang ${ }^{\mathrm{c}}$ Zhen-Hua Zeng ${ }^{d}$ Qiong Li ${ }^{\mathrm{b}}$ \\ Yang Yang $^{\mathrm{b}}$ Ze-Peng Duan ${ }^{\mathrm{b}}$ Yu-Jing Wang ${ }^{\mathrm{b}}$ Yu-Hang $\mathrm{Ai}^{\mathrm{a}}$ \\ ${ }^{a}$ Department of Intensive Care Unit, Xiangya Hospital, Central South University, Changsha, PR China; ${ }^{\mathrm{b}}$ Department \\ of Critical Care Medicine, the First People's Hospital of Chenzhou, Chenzhou, PR China; ' Department of Urology, \\ Huashan Hospital, Fudan University, Shanghai, PR China; 'Department of Critical Care Medicine, Nanfang Hospital, \\ Southern Medical University, Guangzhou, PR China
}

\section{Keywords}

Mitochondrial transcription factor A - Sepsis - Acute kidney injury · Mitochondrial DNA · Toll-like receptor 4 · P38MAPK

\footnotetext{
Abstract

Background: Mitochondrial transcription factor A (TFAM) plays multiple pathophysiologic roles in mitochondrial DNA ( $m$ tDNA) maintenance. However, the role of TFAM in sepsisinduced acute kidney injury (AKI) remains largely unknown. Methods: Lipopolysaccharide (LPS) treatment of HK-2 cells mimics the in vitro model of AKI inflammation. pcDNA3.1 plasmid was used to construct pcDNA3.1-TFAM. shTFAM-543, sh-TFAM-717, sh-TFAM-765, sh-TFAM-904 and pcDNA3.1-TFAM were transfected into HK-2 cells using Lipofectamine 2000. MtDNA transcriptional levels were detected by quantitative real-time polymerase chain reaction (qRTPCR). 3-(4,5)-dimethylthiahiazo (-z-y1)-3,5-di-phenytetrazoliumromide (MTT) assay was performed to assess the cell viability. Changes in reactive oxygen species (ROS) and mito-
}

chondrial membrane potential in HK-2 cells were detected using the corresponding kits. Immunofluorescence experiment was used to investigate the displacement of TFAM. mRNA and protein expression levels of TFAM and its related genes were measured by qRT-PCR and western blot respectively. Mice in sepsis were administered cecal ligation and puncture surgery. Results: LPS treatment was a non-lethal influencing factor, leading to the upregulation of ROS levels and downregulation of mtDNA copy number and NADH dehydrogenase subunit-1 (ND1) expression, and caused damage to the mitochondria. As the LPS treatment time increased, TFAM was displaced from the periphery of the nucleus to cytoplasm. TFAM reduced ROS and P38MAPK levels by inhibiting toll-like receptor 4 (TLR4) expression, ultimately inhibiting inflammation and repairing mtDNA. Conclusions: Our results indicate that TFAM repairs mtDNA by blocking the TLR4/ROS/P38MAPK signaling pathway in inflammatory cells, thereby repairing septic tubular epithelial cells, and TFAM may serve as a new target for sepsis therapy.

(c) 2019 S. Karger AG, Basel

\section{KARGER}

E-Mail karger@karger.com

www.karger.com/pat
(C) 2019 S. Karger AG, Basel Dr. Yu-Hang Ai

Department of Intensive Care Unit, Xiangya Hospital

Central South University, No. 87, Xiangya Road

Changsha 410078, Hunan Province (PR China)

E-Mail ayhicu1978@126.com 


\section{Introduction}

Sepsis is a systemic inflammatory response syndrome caused by infection. It can be secondary to septic shock and multiple organ dysfunction syndrome. It is the leading cause of death in patients with intensive care unit [1-3]. Acute kidney injury (AKI) is one of the most common complications of sepsis. Kidney damage will lead to environmental disorders in the body, which in turn will induce or aggravate the damage of other organs of the body, leading to multiple organ dysfunction syndrome [3]. Although AKI has high morbidity and mortality in patients with sepsis, the mechanism of AKI caused by sepsis remains unclear. Therefore, studying the pathophysiological changes of renal injury in patients with sepsis and finding new effective methods for the treatment of sepsis AKI is a difficult problem we need to study.

Mitochondria are a crucial cell organelles for oxidative phosphorylation and energy synthesis. Oxidative stress is a significant mechanism of inflammatory response, and mitochondria are the main organelle of reactive oxygen species (ROS) production, so mitochondria are closely related to inflammation [4]. Increasing evidences indicated that mitochondrial damage plays a key role in the pathogenesis of AKI [5-7]. The integrity of mitochondrial DNA (mtDNA) plays a crucial role in maintaining mitochondrial function and tissue, as well as cell survival [8, 9]. When AKI occurs, mitochondria in the proximal tubule cells are swollen and broken, leading to disordered arrangement of mitochondria, increased ROS and release of mtDNA [10]. MtDNA release is closely related to the main site of ROS production, and mtDNA is structurally lacking histone modification and protection, so mtDNA is highly susceptible to damage [11]. A growing number of studies have shown that mtDNA lesions and dysfunction may be involved in the development and progression of sepsis-induced AKI $[5,6]$. Therefore, the ability and stability to control mtDNA lesions is expected to be a new strategy for the prevention and treatment of sepsis-induced AKI.

Mitochondrial transcription factor A (TFAM) is one of the regulatory factors regulating mitochondrial replication and transcription, involved in mtDNA transcriptional activation, replication, and copy number regulation [12]. In addition to stimulating mtDNA transcription, TFAM plays a significant pathophysiological role in the maintenance of mtDNA, including the formation of nuclear-like structures, mtDNA copy number stabilization, and preferential recognition of damaged $\mathrm{mtD}$ -
NA $[13,14]$. TFAM overexpression inhibits mitochondrial ROS production and reduces oxidative stress to mtDNA $[15,16]$. However, the relationship between TFAM and AKI-induced mtDNA lesions has rarely been reported.

Based on the above theory, we hypothesized that TFAM can repair sepsis-induced renal tubular epithelial cell injury by inhibiting ROS-induced mtDNA lesions in inflammatory cells. By using gain-of-function and loss-of-function studies, we confirmed the ROS-induced mtDNA lesion suppressor function of TFAM both in vitro and in vivo. It is intended to reveal the molecular mechanisms involved in inflammation-induced AKI and to find new targets for the treatment of sepsis.

\section{Materials and Methods}

\section{Cell Culture and Lipopolysaccharide Treatment}

The human renal proximal convoluted tubule epithelial cell line HK-2 was purchased from American Type Culture Collection, USA. In the lipopolysaccharide (LPS)-group, cells were cultured in Dulbecco's Modified Eagle's Medium (Invitrogen, Gaithersburg, MD, USA) supplemented with $10 \%$ fetal bovine serum (Invitrogen, Gaithersburg, MD, USA) and $4 \mu \mathrm{g} / \mathrm{mL}$ LPS (SigmaAldrich, St. Louis, MO, USA) at $37^{\circ} \mathrm{C}$ for 6,16 and $24 \mathrm{~h}$ respectively. In the control group, cells were cultured in Dulbecco's Modified Eagle's Medium (with 10\% fetal bovine serum) without LPS at $37^{\circ} \mathrm{C}$.

\section{MTT and ROS Assay}

For MTT assay, HK-2 cells were seeded into 96-well plates $(1 \times$ $10^{3}$ cells $\left./ \mu \mathrm{L}\right)$. In the LPS-group, $4 \mu \mathrm{g} / \mathrm{mL}$ LPS was added in to the culture medium and cultured at $37^{\circ} \mathrm{C}$ for 6,16 and $24 \mathrm{~h}$. In the control group, cells were treated without LPS. $5 \mathrm{mg} / \mathrm{mL}$ MTT (Sigma-Aldrich) reagent $(20 \mu \mathrm{L})$ were added into each well and incubated for $4 \mathrm{~h}$. The culture solution was removed and $150 \mu \mathrm{L}$ dimethyl sulfoxide was added into each well. The absorbance at $570 \mathrm{~nm}\left(\mathrm{~A}_{570}\right)$ was measured.

For ROS assay, the ROS detection reagent kit (Nanjing Jiancheng Bioengineering Research Institute, Nanjing, Jiangsu, China) was used in this study to detect the ROS levels according to the manufacturer's instructions.

Gene Knockdown and Overexpression

For gene knockdown, sh-TFAM-543 (5'-ggcaagttgtccaaagaaacc- $\left.3^{\prime}\right)$, sh-TFAM-717 ( $5^{\prime}$-ggcggagtggcaggtatataa- $\left.3^{\prime}\right)$, shTFAM-765 (5' -gctaactccaagtcagattat- $\left.3^{\prime}\right)$, and sh-TFAM-904 (5' ${ }^{\prime}$-gctgaaagattccaagaagct- $\left.3^{\prime}\right)$ were purchased from Youcheng Biotechnology (Changsha, Hunan, China) and transfected into HK2 cells by using Lipofectamine 2000 (Invitrogen, Gaithersburg, MD, USA) according to the manufacturer's instruction. For gene overexpression, pcDNA3.1 (Youcheng Biotechnology) plasmid was used in this study to construct pcDNA3.1-TFAM. The recombinant plasmid was transfected into HK2 cells using Lipofectamine 2000 (Invitrogen). 
Quantitative Real-Time-Polymerase Chain Reaction Analysis

The total RNA of cells was extracted using Trizol reagent (Life technologies, Carlsbad, CA, USA). Reverse transcription was performed according to the instructions of PrimeScript RT reagent Kit with gDNA Eraser (Takara, Dalian, China). Quantitative realtime polymerase chain reaction (qRT-PCR) was performed using the SYBR Green qPCR Master Mix Kit (Takara, Dalian, China). The reaction conditions were as follows: $96^{\circ} \mathrm{C} 6 \mathrm{~min}$, followed by 40 cycles each at $96^{\circ} \mathrm{C} 15 \mathrm{~s}, 57^{\circ} \mathrm{C} 30 \mathrm{~s}, 72^{\circ} \mathrm{C} 30$ s. $\beta$-actin or $\beta$-globin was used as an internal standard and CT method was used to calculate the relative expression level. The primers were shown in Table 1.

\section{Western Blotting Assay}

Cells were lysed by RIPA lysis and extraction buffer (Thermo Fischer Scientific, Waltham, MA, USA) and then centrifuged to obtain the supernatant. Protein samples $(50 \mu \mathrm{g})$ were electrophoresed on $10 \%$ SDS-PAGE, transferred to PVDF membrane, and blocked in 5\% non-fat milk for $2 \mathrm{~h}$ at room temperature. Then, incubated with the primary antibodies at $4{ }^{\circ} \mathrm{C}$ overnight, followed by application of secondary antibodies $(1: 10,000$ dilution; ProteinSimple, San Jose, CA, USA). The protein expression levels were measured by an enhanced chemiluminescence detection kit (Thermo). The antibodies used in this study included anti-TFAM (1:2,000 dilution; Cell Signaling, Danvers, MA, USA), anti- $\beta$-actin (1:2,000 dilution; Cell Signaling).

\section{Mitochondrial Membrane Potential Detection}

The cells were seeded into a 12 -well plate with $2.0 \times 10^{6}$ cells/ $\mathrm{mL}$, and treated with LPS $(4 \mu \mathrm{g} / \mathrm{mL})$ for 6,16 and $24 \mathrm{~h}$, respectively. The detection of mitochondrial membrane potential was performed using the mitochondrial membrane potential assay kit with JC-1 (Beyotime Biotechnology, Shanghai, China) according to the manufacturer's instructions. Finally, the samples were observed and photographed with a fluorescence microscope.

\section{Immunofluorescence Staining}

Cells were seeded into a 12 -well plate with $2.0 \times 10^{6}$ cells $/ \mathrm{mL}$ and cultured for $24 \mathrm{~h}$, then cells were stimulated with LPS (100 ng/ $\mathrm{mL}$ ) for $4 \mathrm{~h}$. After washing with PBS buffer for 3 times, the cells were fixed with $4 \%$ paraformaldehyde for $15 \mathrm{~min}$. Treated with $0.5 \%$ Triton- 10 for 20 min and then blocked with $10 \%$ goat serum for $30 \mathrm{~min}$. Incubated with primary antibodies (anti-TFAM; 1:500 dilution; Cell Signaling) at $4^{\circ} \mathrm{C}$ overnight. After washing with PBS buffer for 3 times, the cells were incubated with secondary antibodies (1:2,000 dilution; Cell Signaling) at $37^{\circ} \mathrm{C}$ for $1 \mathrm{~h}$. The nuclei was stained with DAPI for $5 \mathrm{~min}$. An appropriate amount of anti-fluorescence quencher was added. Finally, it was observed under a fluorescence microscope.

\section{Preparation of Sepsis Model}

C57BL/6J mice were purchased from the fourth military medical university model animal center (Xian, Shanxi, China). All mice were fasted for $12 \mathrm{~h}$ before the model preparation experiment. $0.5 \mathrm{~mL} / \mathrm{kg}$ chloral hydrate (7\%) was used for intraperitoneal injection of general anesthesia. The abdomen was opened under aseptic conditions and the cecum was separate. Then, ligation was performed at $2 \mathrm{~cm}$ from the end. Perforation was performed at the end of the cecum and a small amount of feces was squeezed. Finally,
Table 1. Primers used for qRT-PCR analysis

\begin{tabular}{ll}
\hline Genes & Primer sequences (5'-3') \\
\hline TFAM & F: 5'-CCGAGGTGGTTTTCATCTGT-3' \\
& R: 5'-CCGCCCTATAAGCATCTTGA-3' \\
\hline P38-MAPK & F: 5'-TATGCGTCTGACAGGAACACC-3' \\
& R: 5'-GATCGGCCACTGGTTCATCA-3' \\
\hline TLR4 & F: 5'-AGAAACTGCTCGGTCAGACG-3' \\
& R: 5'-AATGGAATCGGGGTGAAGGG-3' \\
\hline MAPK & F: 5'-ATTACGACCCGAGTGACGAG-3' \\
& R: 5'-CGTCTTCAGAGACAGCCAGGAG-3' \\
\hline ND1 & F: 5'-CCCTAAAACCCGCCACATCT-3' \\
& R: 5'-GAGCGATGGTGAGAGCTAAGGT-3' \\
\hline$\beta$-Actin & F: 5'-GCAGGAGTACGATGAGTCCG-3' \\
& R: 5'-ACGCAGCTCAGTAACAGTCC-3' \\
\hline$\beta$-Globin & F: 5'-AAAGGTGCCCTTGAGGTTGTC-3' \\
& R: 5'-TGAAGGCTCATGGCAAGAAA-3'
\end{tabular}

TFAM, Mitochondrial transcription factor A; qRT-PCR, quantitative real-time polymerase chain reaction.

the cecum was put back into the abdominal cavity and the abdomen was closed layer by layer. In the control group, ligation and perforation were not performed when the cecum end was isolated. Mice were sacrificed 24, 48, and $72 \mathrm{~h}$ after surgery.

\section{Hematoxylin-Eosin, Masson, and Immunohistochemistry}

Staining

The mouse kidney tissues were fixed in $4 \%$ paraformaldehyde for $24 \mathrm{~h}$ and then dehydrated with 100, 95, 80, and 70\% ethanol. Subsequently, the samples were embedded in paraffin and sectioned in $3 \mu \mathrm{m}$ thickness. For Hematoxylin-Eosin (H\&E) staining assay, the thicknesses were stained in hematoxylin for $5 \mathrm{~min}$ and in eosin for $30 \mathrm{~s}$. For Masson staining, Trichrome Stain (Masson) Kit (Sigma, MO, USA) was used according to the manufacturer's instruction. For Immunohistochemistry (IHC) staining, antigen retrieval was performed by the heat repair method. The thicknesses were soaked in $3 \% \mathrm{H}_{2} \mathrm{O}_{2}$ for 10 min and blocked with nonimmune goat serum at room temperature for $10 \mathrm{~min}$. Then, the thicknesses were incubated with primary antibodies (anti-TNF- $\alpha$; 1:500 dilution; Cell Signaling) at $4{ }^{\circ} \mathrm{C}$ overnight, followed by incubation with secondary antibodies (1:2,000 dilution; Cell Signaling) at room temperature for $30 \mathrm{~min}$. Subsequently, the thicknesses were stained with DAB reagent (Sigma) for $5 \mathrm{~min}$, and then stained with hematoxylin for 2 min. Finally, the histomorphological changes were observed under the microscope (LEICADMLB2, Germany).

\section{Statistical Analysis}

SPSS 22.0 statistical software version 22.0 (SPSS, Chicago, IL, USA) was used in this study and all experiments were repeated in 3 times. Data were expressed as mean \pm SD. Student $t$ test was performed to compare different groups. A $p$ value $<0.05$ was accepted as statistically significant difference. 


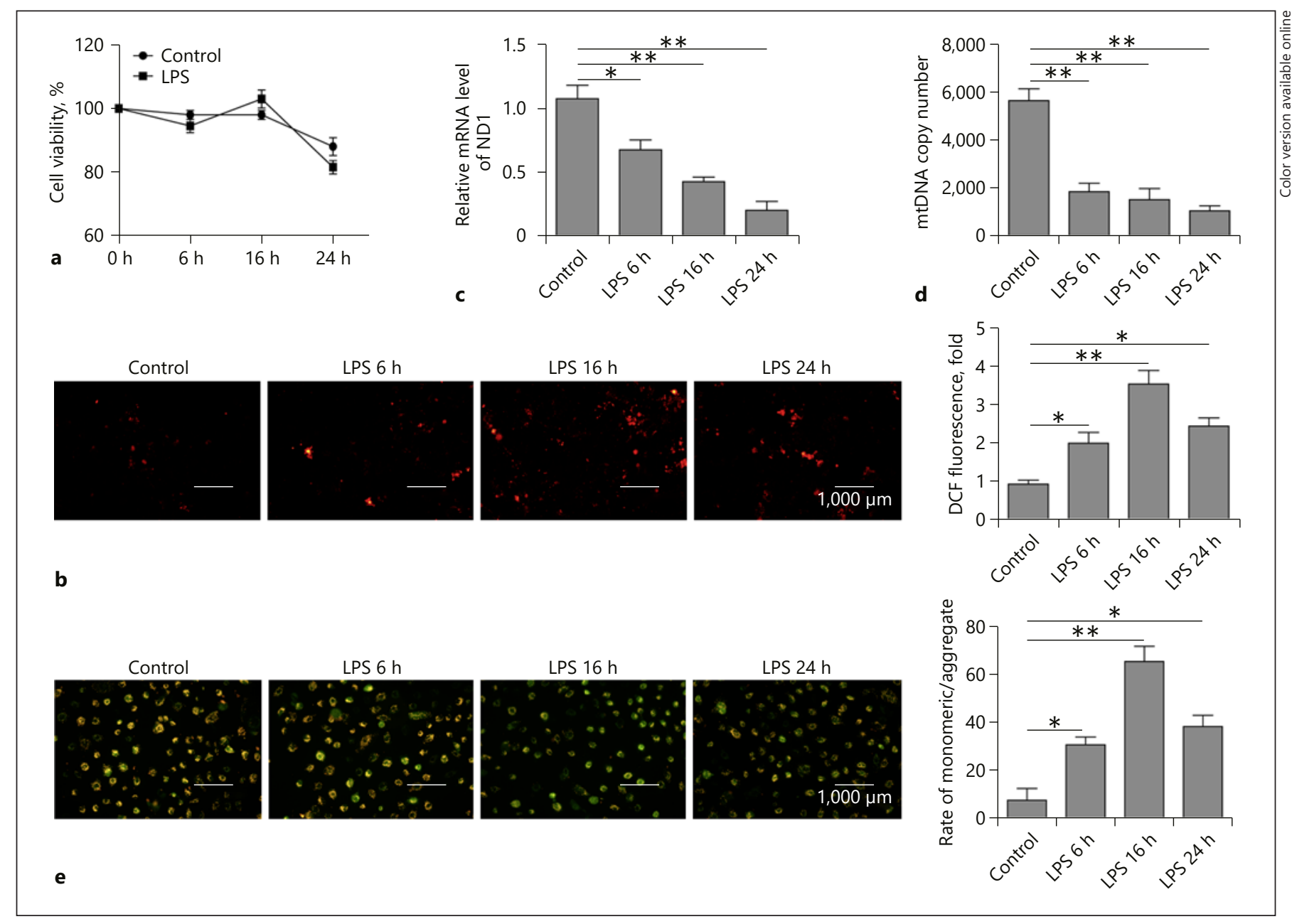

Fig. 1. Effects of LPS treatment on mitochondria in HK-2 cells. For LPS-group, HK-2 cells were cultured in DMEM (with 10\% FBS) and treated with LPS $(4 \mu \mathrm{g} / \mathrm{mL})$ for 6,16 , and $24 \mathrm{~h}$. For the control group, HK-2 cells were cultured in DMEM (with 10\% FBS) without LPS. a MTT assay was performed in both LPS-group cells and the control group cells. b ROS levels were measured in both LPSgroup cells and the control group cells. c Quantitative RT-PCR

\section{Results}

\section{Effects of LPS Treatment on Mitochondria in HK-2} Cells

To explore the effects of LPS on mitochondrial cells, we treated HK-2 cells with $4 \mu \mathrm{g} / \mathrm{mL}$ of LPS for 6,16 , and $24 \mathrm{~h}$. MTT assay was performed in this study to assess the cell viability. As shown in Figure 1a, there was no significant difference between control cells and LPS-treated cells $(p>0.05)$, indicating that LPS was a non-lethal factor in HK-2 cells. However, the treatment of LPS resulted in a significant increase in intracellular ROS levels, with the analysis of relative ND1 expression levels in both LPS-group cells and the control group cells. d Quantitative RT-PCR analysis of mtDNA levels in both LPS-group cells and the control group cells. e Mitochondrial membrane potential detection was performed in both LPS-group cells and the control group cells. ${ }^{*} p<0.05$ and ${ }^{* *}$ $p<0.01$ were considered to indicate a statistically significant difference. LPS, lipopolysaccharide.

most significant upregulation of LPS treatment for $16 \mathrm{~h}$ (Fig. 1b). The mitochondrially encoded ND1, as an important component of respiratory chain complex I, is involved in the key steps of oxidative phosphorylation to produce energy, and its abnormal expression indicates mitochondrial dysfunction. Therefore, we performed the qRT-PCR experiment to measure the relative mRNA expression levels of ND1 and the mtDNA copy number. As shown in Figure 1c and d, compared with the control cells, LPS treatment resulted in a significant decrease in both the relative expression levels of ND1 $(p<0.05)$ and mtDNA copy number $(p<0.05)$. Furthermore, LPS treat- 


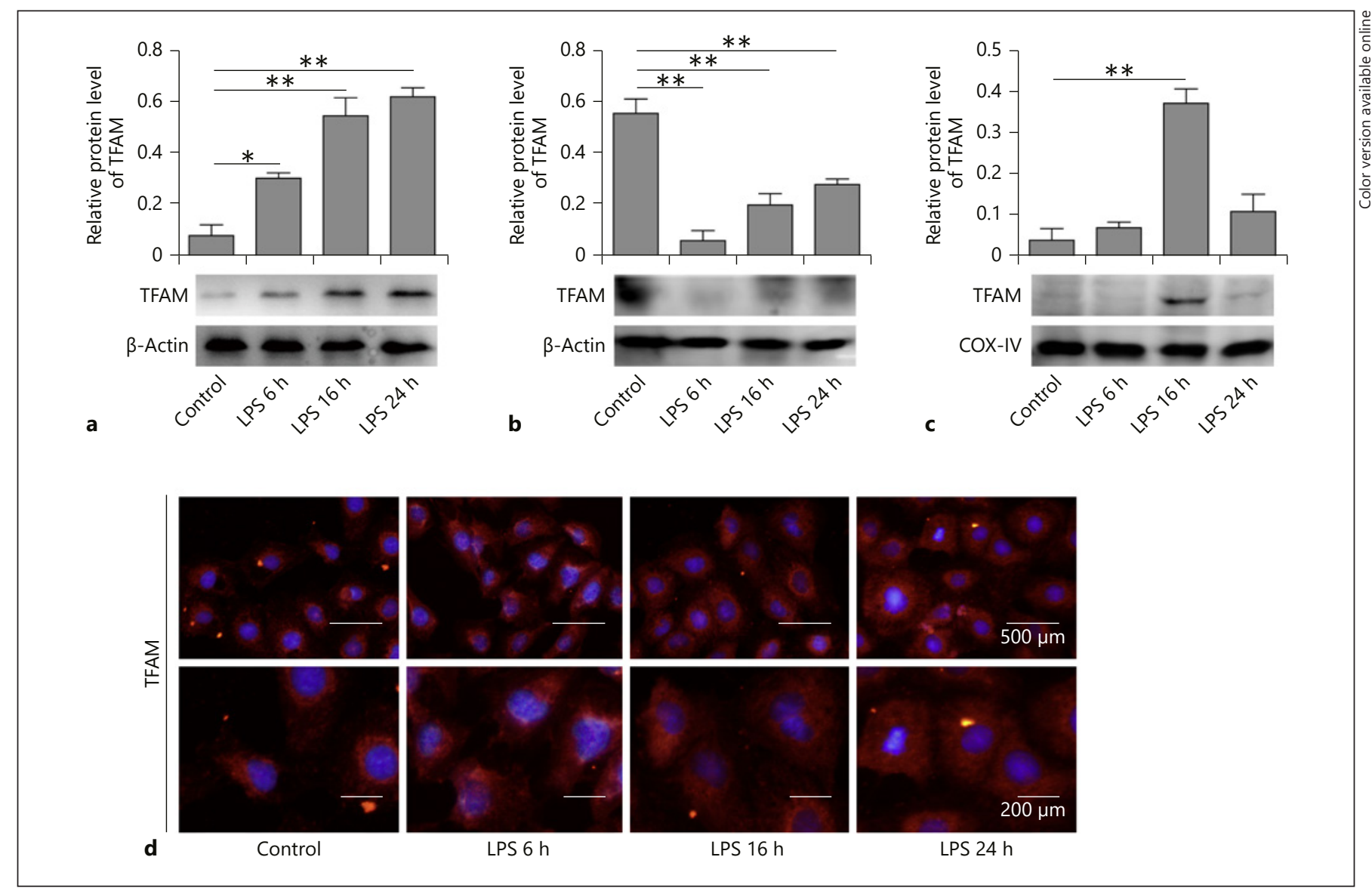

Fig. 2. Effects of LPS treatment on TFAM levels in HK-2 cells. a Detection of TFAM expression level in total protein by western blotting assay. b Detection of TFAM expression levels in cytoplasm by western blotting assay. c Detection of TFAM expression levels in mitochondria by western blotting assay. $\mathbf{d}$ Immunofluo- rescence staining was performed to observe the expression changes and displacement of TFAM after LPS treatment. ${ }^{*} p<0.05$ and ** $p<0.01$ were considered to indicate a statistically significant difference. LPS, lipopolysaccharide; TFAM, mitochondrial transcription factor A; COX, cytochrome c oxidase. ment changed the mitochondrial membrane potential, leading to mitochondrial pathway-dependent early apoptosis in HK-2 cells (Fig. 1e). Taken together, LPS treatment was a non-lethal influencing factor, leading to the upregulation of ROS levels and downregulation of $\mathrm{mtD}$ NA copy number and ND1 gene expression, and caused damage to the mitochondria.

\section{Effects of LPS Treatment on TFAM Levels in HK-2 Cells}

To further assess the effects of LPS on mitochondrial, we performed the western blotting assay to measure TFAM levels in total protein of cells, cytoplasm and mitochondria. As shown in Figure 2a, compared with the control group, LPS treatment increased TFAM levels in total protein. Compared with the control cells, LPS treat- ment for $6 \mathrm{~h}$ resulted in a significant decrease in TFAM levels in the cytoplasm $(p<0.05)$, while LPS treatment for 16 and $24 \mathrm{~h}$ significantly increased the TFAM levels in the cytoplasm ( $p<0.05$; Fig. $2 b)$. Furthermore, compared with control cells, LPS treatment significantly increased the expression levels of TFAM in mitochondria, and the upregulation folds were highest in LPS treatment for $16 \mathrm{~h}$ $(p<0.05$; Fig. $2 c)$. In addition, we used immunofluorescence experiments to investigate the displacement of TFAM in both control cells and LPS-treated cells. The results indicated that, as the LPS treatment time increased, TFAM was displaced from the periphery of the nucleus to the cytoplasm (Fig. 2d). Taken together, LPS treatment caused significant changes in the expression and localization of TFAM, which may play a role in the mitochondrial changes caused by LPS. 
Effects of TFAM Overexpression and Knockdown in

\section{HK-2 Cells}

To explore the role of TFAM in HK-2 cells, TFAM was effectively overexpressed and knocked down in HK-2 cells. As shown in Figure 3a, compared with the control cells, transfected with pcDNA3.1-TFAM plasmid resulted in a significant increase in the expression levels of TFAM $(p<0.05)$. As expected, compared with the control cells, TFAM levels were significantly lower in HK-2 cells, which transfected with sh-TFAM-543, shTFAM-717, sh-TFAM-765, and sh-TFAM-904 $(p<0.05$; Fig. 3b). Subsequently, qRT-PCR assay was used to measure the relative expression levels of $\mathrm{ND} 1$ and the $\mathrm{mtD}$ NA copy number after overexpression or inhibition of TFAM. As shown in Figure $3 \mathrm{c}$ and d, overexpression of TFAM resulted in a significant increase in both ND-1 and mtDNA levels $(p<0.05)$; otherwise, TFAM knocked down resulted in a significant decreased in both ND-1 and mtDNA levels $(p<0.05)$. Furthermore, the overexpression of TFAM resulted in a marked decrease in ROS levels, and the LPS-treatment groups had a higher percentage of downregulation than the untreated group $(p<0.05$; Fig. 3e). As expected, knockdown of TFAM resulted in a marked increase in ROS levels, and the groups without LPS treatment had a higher upregulation fold compared with the LPS-treatment groups $(p<0.05$; Fig. 3e). Then, we measured the expression levels of Tolllike receptor 4 (TLR4), MAPK and P38MAPK by qRTPCR experiment. The data indicated that overexpression of TFAM decreased the expression levels of TLR4, MAPK, and P38MAPK, and the change fold of the LPS treatment group was more obvious than that of the untreated group ( $p<0.05$; Fig. 3f). And knockdown of TFAM caused the opposite changes ( $p<0.05$; Fig. 3f). Taken together, these results suggested TFAM reduced ROS and P38MAPK levels by inhibiting the expression of TLR4, ultimately inhibiting inflammation and repairing mtDNA.

\section{TFAM Involved in the Regulation of the Repair of}

Sepsis Induced AKI in vivo

To further explore the role of TFAM in sepsis-induced AKI, the cecal ligation and puncture (CLP) model mice were prepared. H\&E, Masson and IHC staining assays were performed $24 \mathrm{~h}$ after surgery in both CLP model group and control group. The results of $\mathrm{H} \& \mathrm{E}$ and Masson staining assays indicated there was no obvious pathological change in the control group, while inflammatory cell infiltration and renal fibrosis were observed in sepsis model mice (Fig. 4a). Furthermore, IHC staining assay indicated that the expression of TNF- $\alpha$ was enhanced in sepsis model mice (Fig. 4a). The results of staining assays displayed that the mouse sepsis model was successfully constructed, and inflammatory infiltration was observed in the mouse kidney tissues.

Mice in CLP model group were sacrificed 24, 48, and $72 \mathrm{~h}$ after surgery, and the levels of mtDNA, ND1, TFAM, ROS, TLR4, MAPK, and P38MAPK were measured in this study. As shown in Figure $4 \mathrm{~b}$ and c, mtDNA and ND1 levels of sepsis model mice were decreased and then increased with time compared with control team. The results of western blotting assay indicated that the TFAM levels were increased in model mice (Fig. 4d). Furthermore, the ROS levels of sepsis model mice were increased and then decreased with time too (Fig. 4e). Finally, we used the qRT-PCR experiment to measure the expression levels of TLR4, MAPK, and P38MAPK. As shown in Figure $4 \mathrm{f}$, the relative expression levels of TLR4, MAPK, and P38MAPK were increased and then decreased in sepsis model mice.

Collectively, above results further indicated that TFAM inhibited ROS and P38MAPK by inhibiting TLR4, thereby inhibiting inflammation and repairing mtDNA (Fig. 5). The trends of animal experiments were consistent with those of cell experiments.

\section{Discussion}

Sepsis-induced AKI is a crucial type of AKI, accounting for approximately $50 \%$ of all acute kidney injuries [17]. However, at present there is almost no specific and effective means to prevent sepsis-induced AKI clinically. In recent years, despite the deepening of research on sepsis, the occurrence and death of sepsis have not improved significantly [2]. In the treatment of sepsis, antibiotics, fluid resuscitation, mechanical ventilation and other treatments have a certain effect, but the application of glucocorticoids, glucose control and kidney replacement has been controversial [18-20]. Studying the mechanism of sepsis-induced AKI development and progression can provide ideas and clues to solve this clinical problem. Recent studies have shown that mitochondrial biosynthesis is involved in the recovery of mitochondrial damage in organs during sepsis, thereby improving the prognosis of sepsis [21-24]. Mitochondria participate in a wide range of innate immune pathways that contribute to cellular damage and innate immune activation [25, 26]. This study investigated the protective effect of TFAM on sepsis-induced AKI from the perspective of mtDNA lesions. 

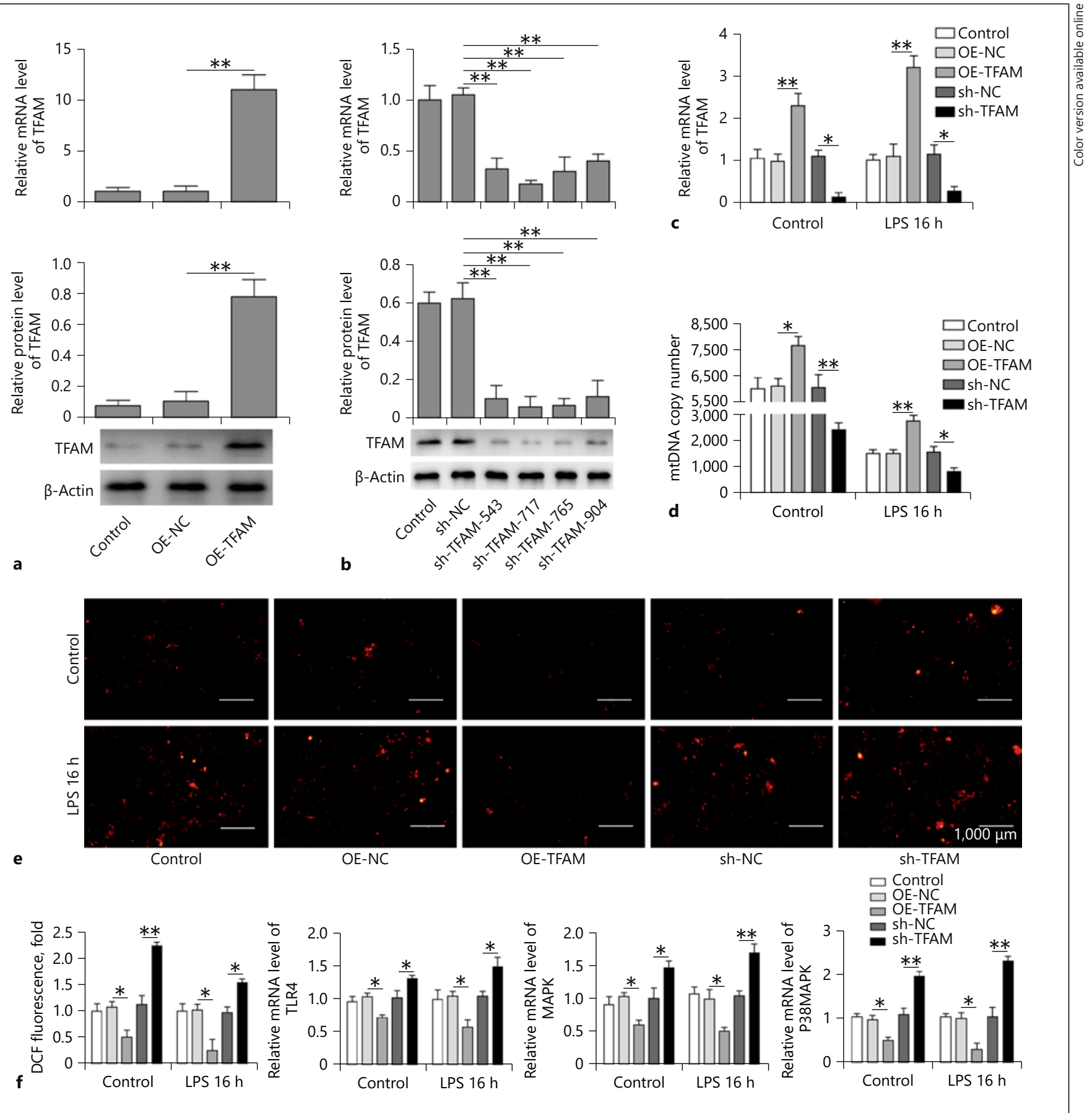

e

OE-NC

OE-TFAM

sh-NC

sh-TFAM

$\square$ Control OE-NC
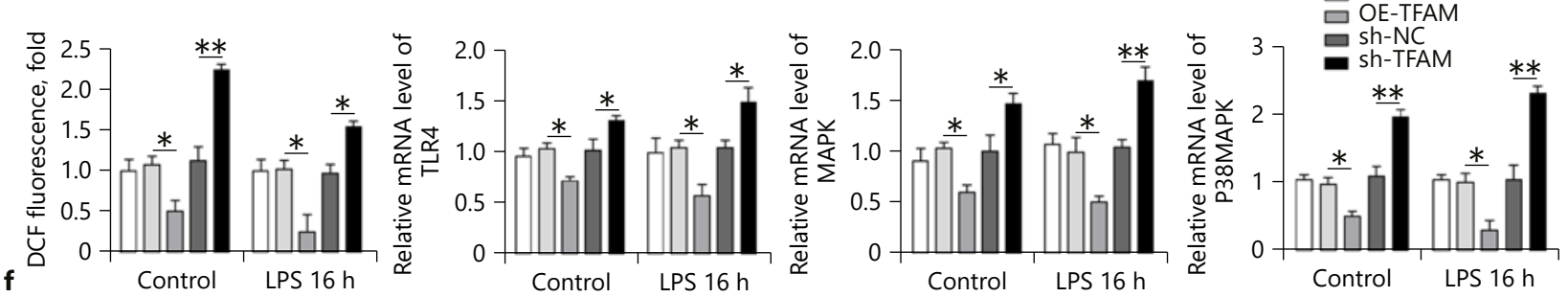

Fig. 3. Effects of TFAM overexpression and knockdown in HK-2 cells. a Quantitative RT-PCR and western blotting assays were performed to measure the TFAM levels in HK2 cells transfected with TFAM overexpression plasmid. b Quantitative RT-PCR and western blotting assays were performed to measure the TFAM levels in HK2 cells transfected with sh-TFAM. c Detection of ND1 expression levels by quantitative RT-PCR in both LPS-group cells and the control group cells transfected with TFAM overexpression plasmid or sh-TFAM. d Detection of mtDNA levels by quantitative RT-PCR in both LPS-group cells and the control group cells trans- fected with TFAM overexpression plasmid or sh-TFAM. e Detection of ROS levels in both LPS-group cells and the control group cells transfected with TFAM overexpression plasmid or sh-TFAM. f Detection of TLR4, MAPK and P38MAPK expression levels by quantitative RT-PCR in both LPS-group cells and the control group cells transfected with TFAM overexpression plasmid or shTFAM. ${ }^{*} p<0.05$ and ${ }^{* *} p<0.01$ were considered to indicate a statistically significant difference. LPS, lipopolysaccharide; TFAM, mitochondrial transcription factor A. 

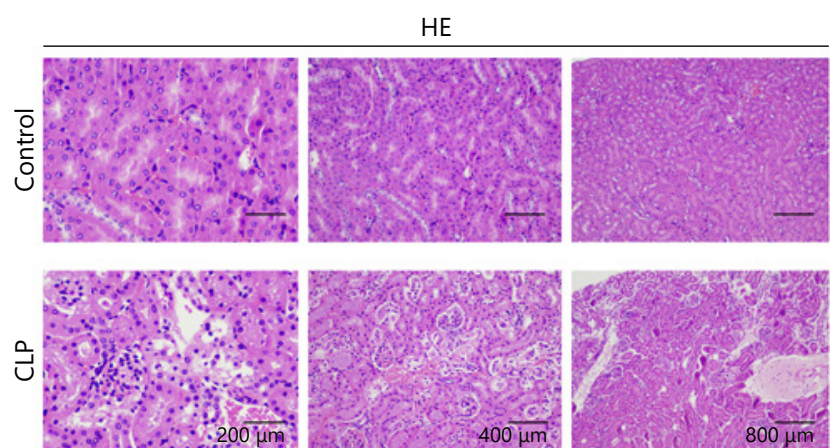

$800 \mu \mathrm{m}$
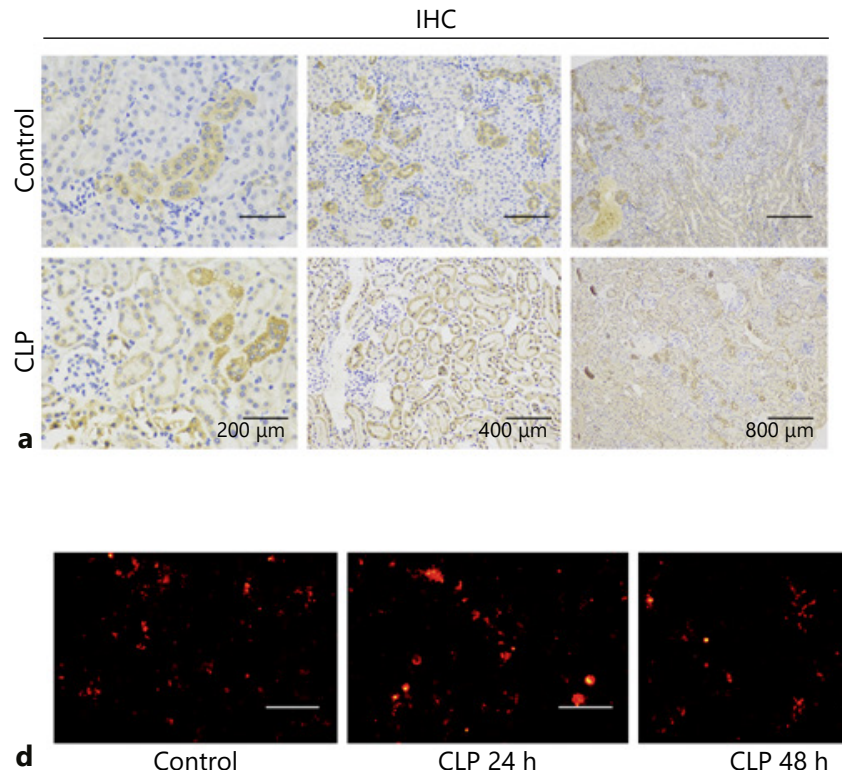

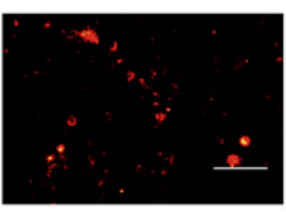

CLP $24 \mathrm{~h}$

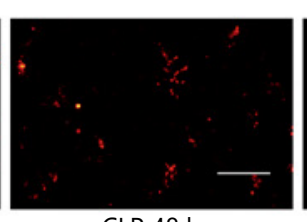

CLP $48 \mathrm{~h}$
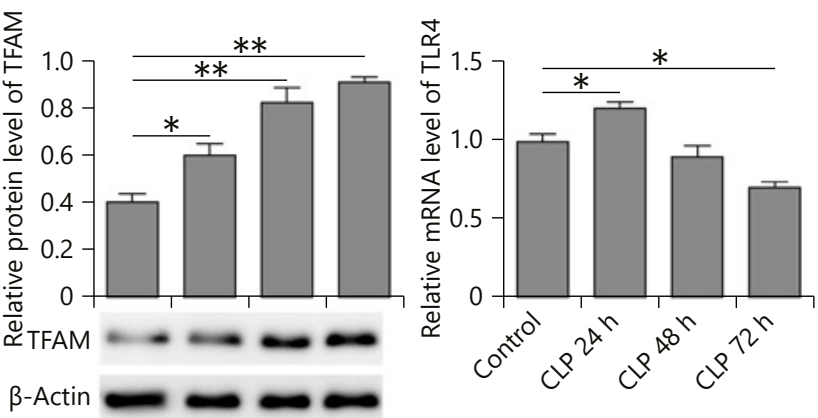

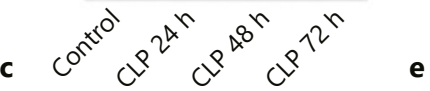
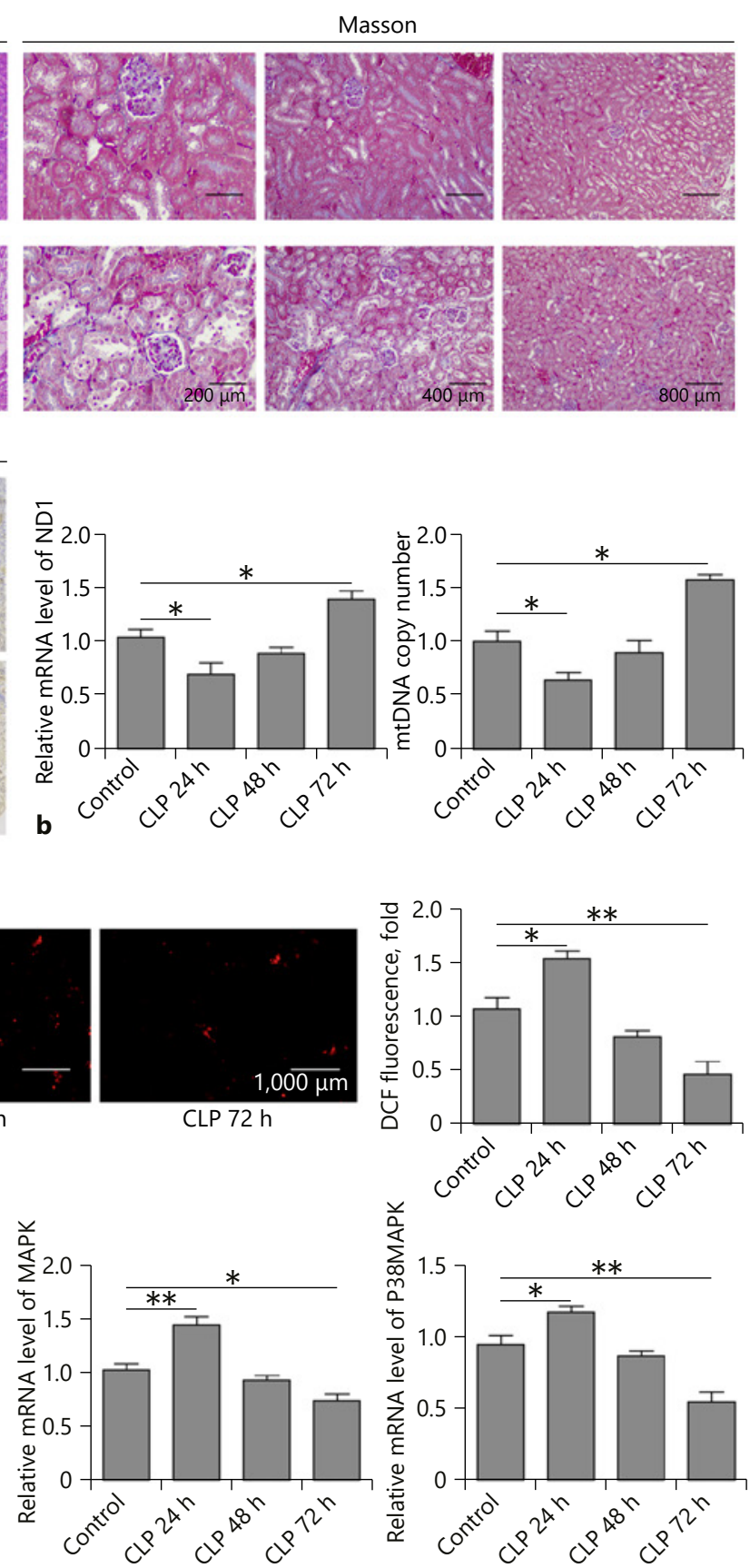

CLP $72 \mathrm{~h}$
Fig. 4. TFAM involved in the regulation of the repair of renal tubular epithelial cells in sepsis in vivo. Mice in sepsis model group were sacrificed 24, 48, and $72 \mathrm{~h}$ after surgery. a H\&E, Masson and IHC staining assays were performed $24 \mathrm{~h}$ after surgery in both sepsis model group and control group. $\mathbf{b}$ qRT-PCR analysis of mtDNA and ND1 levels in both sepsis model group and control group. c Western blotting analysis of TFAM levels in both sepsis model group and control group. $\mathbf{d}$ ROS levels were measured in both the sepsis model group and control group. e qRT-PCR analysis of TLR4, MAPK and P38MAPK expression levels in both the sepsis model group and control group. ${ }^{*} p<0.05$ and ${ }^{* *} p<0.01$ were considered to indicate a statistically significant difference. CLP, cecal ligation and puncture; IHC, Immunohistochemistry; TFAM, mitochondrial transcription factor $\mathrm{A}$. 


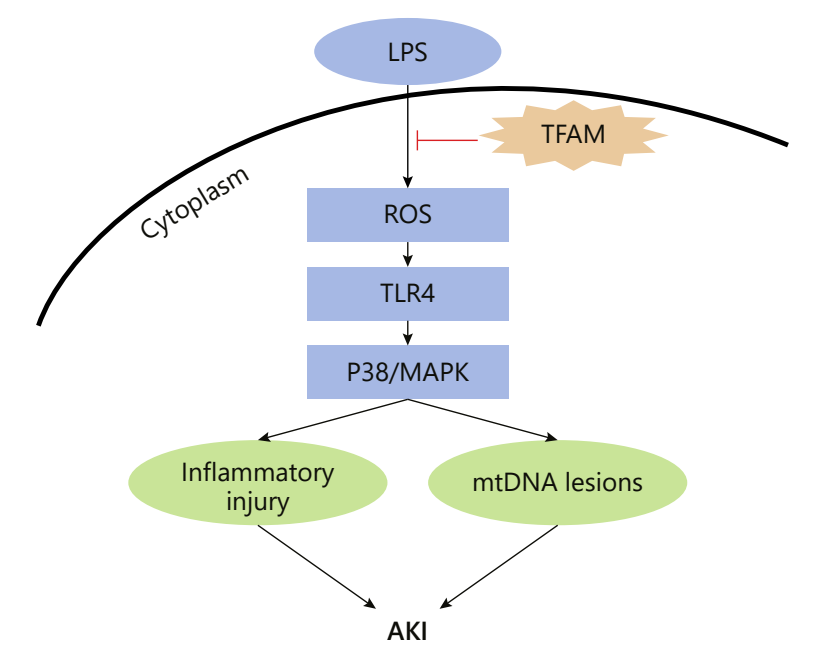

Fig. 5. The proposed mechanism of TFAM-mediated the repairment of renal tubular epithelial cells in sepsis. TFAM alleviated inflammation and repaired mtDNA by inhibiting ROS-mediated P38MAPK signaling by reducing TLR4 expression. ROS, reactive oxygen species; TLR4, toll-like receptor 4; DNA, mtDNA, mitochondrial DNA; TFAM, mitochondrial transcription factor A; LPS, lipopolysaccharide; AKI, acute kidney injury.

MtDNA plays a significant role in maintaining mitochondrial homeostasis. However, mtDNA is highly susceptible to oxidative stress due to its proximity to the mitochondrial inner membrane of the respiratory chain and its lack of intron and damage repair capabilities [27, 28]. Increasing oxidative stress to mtDNA will damage electron transport, increase ROS production, and further lead to more mtDNA lesions. In a variety of mitochondriarelated diseases, primary or secondary damage to mtDNA will lead to mitochondrial dysfunction, including energy metabolism disorders, oxidative phosphorylation abnormalities, and mitochondrial oxidative stress [29]. Studies have confirmed that sepsis causes changes in mitochondrial permeability, loss of mitochondrial membrane potential, mitochondrial swelling, and mitochondrial matrix coagulation and vacuolization in severe cases, leading to impaired mitochondrial function [30]. This study showed that after receiving pro-inflammatory stimuli, HK2 cells significantly upregulated ROS expression, downregulated mtDNA expression and disrupted mitochondria, which is consistent with previous reports [31].

It has been previously thought that mtDNA is considered to be naked due to the lack of histones [32]. But there is growing evidence that mtDNA may not be naked but has a special spatial structure in which TFAM plays a cru- cial role in this structure [33]. TFAM protein contains 2 HMGbox domains, belonging to the typical high migration protein family, which has the function of binding, wrapping, winding mtDNA and unwinding [34]. In addition, TFAM entangled mtDNA to form a stable nuclear-like structure. In this nuclear-like structure, TFAM is similar to the action of histones in the genome. Studies have shown that mtDNA is prone to mutations, fragment deletions, and degradation if mtDNA nuclear-like structures are destabilized [35]. Moreover, the regulation of $\mathrm{mtDNA}$ replication and transcription is also dependent on the stability of the mtDNA nuclear-like structure [35]. Overexpression of TFAM promotes mtDNA lesion repair. TFAM can preferentially bind to damaged mtDNA and regulate the excision repair of bases $[36,37]$. Studies have shown that overexpression of TFAM plays a protective role rather than a transcription factor in animal models of aging and myocardial infarction associated with oxidative stress to mtDNA $[38,39]$. This study demonstrates the presence of $\mathrm{mtDNA}$ lesions in sepsis-induced AKI. Importantly, mtDNA lesions further aggravate mitochondrial oxidative stress by encoding key proteins $\mathrm{ND}-1$ in the respiratory chain. As the pro-inflammatory stimuli time increased, TFAM was displaced from the periphery of the nucleus to the cytoplasm. Moreover, TFAM expression is elevated, repairing mtDNA lesions, and inhibiting cell damage caused by oxidative stress.

The innate immune response is the first step in the inflammatory response to sepsis [40]. TLR transmembrane proteins may act as receptors for signal transduction and are more involved in the process of innate immune responses [41]. It has been reported that TFAM has a common proinflammatory active site as both HMGB1 structural and functional homologs [26]. Therefore, TFAM released from damaged cells can be recognized by the immune system through different TLRs and/or RAGE, which may lead to local and systemic inflammation $[42,43]$. Our study found that overexpression of TFAM after pro-inflammatory stimulation reduced TLR4 expression. It is suggested that TFAM may inhibit inflammation by inhibiting TLR4 expression during the development of sepsis-induced AKI. As a common ligand for bacteria and viruses, TLR activates downstream inflammatory pathways such as MAPKs to induce the release of inflammatory factors such as TNFa, IL6 , and IL- $1 \beta$, thereby exerting its inflammatory effects [44]. Our results indicate that TFAM repairs mtDNA by blocking the TLR4/ROS/P38MAPK signaling pathway. However, this study only explored the role of intracellular TFAM, and the mechanism of action of TFAM release into the extracellular can be used as the next step of inquiry. 
Taken together, our results indicate that TFAM repairs mtDNA by blocking the TLR4/ROS/P38MAPK signaling pathway in inflammatory cells, thereby repairing septic tubular epithelial cells, and TFAM may serve as an effective target for the treatment of sepsis. Exogenously increased TFAM expression may be important for sepsis therapy. It was reported that dietary isoflavone daidzein attenuated mitochondrial damage by increasing TFAM level [45]. Therefore, increasing the expression of TFAM by using some natural compounds intervention may have an ideal therapeutic effect on AKI caused by sepsis.

\section{Acknowledgment}

We would like to give our sincere gratitude to the reviewers for their constructive comments.

\section{Statement of Ethics}

The authors have no ethical conflicts to disclose.

\section{Disclosure Statement}

The authors declare that they have no conflicts of interest to disclose.

\section{Funding Sources}

This work was supported by National Natural Science Foundation of China (No. 81601708) and Natural Science Foundation of Hunan Province, China (No. 2018JJ2014).

\section{Author Contributions}

X.-G.D. designed the study, did literature research, performed experimental studies, prepared and edited the manuscript. T.L., W.-B.H., Z.-H.Z., and Q.L. performed experimental studies. Y.Y., Z.-P.D., and Y.-J.W. acquired and analyzed data. Y.-H.A. designed the study, edited and reviewed the manuscript. All authors approved the submission of this work.

\section{References}

1 Singer M, Deutschman CS, Seymour CW, Shankar-Hari M, Annane D, Bauer M, et al. The Third International Consensus Definitions for Sepsis and Septic Shock (Sepsis-3). JAMA. 2016 Feb;315(8):801-10.

2 Timsit JF, Ruppe E, Ferrer R. Focus on sepsis: new concepts and findings in sepsis care. Intensive Care Med. 2018 Nov;44(11):1997-9.

3 Gómez H, Kellum JA. Sepsis-induced acute kidney injury. Curr Opin Crit Care. 2016 Dec; 22(6):546-53.

4 Suzuki T, Yamaguchi H, Kikusato M, Hashizume O, Nagatoishi S, Matsuo A, et al. Mitochonic Acid 5 Binds Mitochondria and Ameliorates Renal Tubular and Cardiac Myocyte Damage. J Am Soc Nephrol. 2016 Jul;27(7): 1925-32.

5 Yang J, Wu F, Chen J, Yang Y. [Screening of mitochondrial DNA damage repair genes in rats with septic acute kidney injury]. Zhejiang Da Xue Xue Bao Yi Xue Ban. 2018 Jan;47(1): 41-50.

$6 \mathrm{Hu}$ Q. et al. Urinary Mitochondrial DNA Identifies Renal Dysfunction and Mitochondrial Damage in Sepsis-Induced Acute Kidney Injury. Oxid Med Cell Longev 2018, 8074936, doi:https://doi.org/10.1155/2018/8074936. (2018).

7 Cheng W, Zhao F, Tang CY, Li XW, Luo M, Duan SB. Comparison of iohexol and iodixanol induced nephrotoxicity, mitochondrial damage and mitophagy in a new contrast-induced acute kidney injury rat model. Arch Toxicol. 2018 Jul;92(7):2245-57.
8 Bonora M, Pinton P. Mitochondrial DNA keeps you young. Cell Death Dis. 2018 Sep; 9(10):992.

9 Kauppila TE, Bratic A, Jensen MB, Baggio F, Partridge L, Jasper $\mathrm{H}$, et al. Mutations of mitochondrial DNA are not major contributors to aging of fruit flies. Proc Natl Acad Sci USA. 2018 Oct;115(41):E9620-9.

10 Martin L, Thiemermann C. Mitochondrial DNA in Acute Kidney Injury: chicken or Egg? Shock. 2018 Mar;49(3):352-3.

11 Giordano L, Deceglie S, d'Adamo P, Valentino ML, La Morgia C, Fracasso F, et al. Cigarette toxicity triggers Leber's hereditary optic neuropathy by affecting mtDNA copy number, oxidative phosphorylation and ROS detoxification pathways. Cell Death Dis. 2015 Dec;6(12):e2021.

12 Dong X, Ghoshal K, Majumder S, Yadav SP, Jacob ST. Mitochondrial transcription factor $\mathrm{A}$ and its downstream targets are up-regulated in a rat hepatoma. J Biol Chem. 2018 Aug; 293(33): 12947.

13 Kang D, Kim SH, Hamasaki N. Mitochondrial transcription factor A (TFAM): roles in maintenance of mtDNA and cellular functions. Mitochondrion. 2007 Feb-Apr;7(1-2):39-44.

14 Vasileiou PV, Mourouzis I, Pantos C. Principal Aspects Regarding the Maintenance of Mammalian Mitochondrial Genome Integrity. Int J Mol Sci. 2017 Aug;18(8):E1821.

15 Kunkel GH, Chaturvedi P, Tyagi SC. Mitochondrial pathways to cardiac recovery: TFAM. Heart Fail Rev. 2016 Sep;21(5):499-517.
16 Diebold I, Hennigs JK, Miyagawa K, Li CG, Nickel NP, Kaschwich M, et al. BMPR2 preserves mitochondrial function and DNA during reoxygenation to promote endothelial cell survival and reverse pulmonary hypertension. Cell Metab. 2015 Apr;21(4):596608

17 Maiden MJ, Otto S, Brealey JK, Finnis ME, Chapman MJ, Kuchel TR, et al. Structure and Function of the Kidney in Septic Shock. A Prospective Controlled Experimental Study. Am J Respir Crit Care Med. 2016 Sep;194(6): 692-700.

18 Toma A, Stone A, Green RS, Gray S. Steroids for patients in septic shock: the results of the CORTICUS trial. CJEM. 2011 Jul;13(4):273-6.

19 Mesotten D, Gielen M, Sterken C, Claessens K, Hermans G, Vlasselaers D, et al. Neurocognitive development of children 4 years after critical illness and treatment with tight glucose control: a randomized controlled trial. JAMA. 2012 Oct;308(16):1641-50.

20 Chon GR, Chang JW, Huh JW, Lim CM, Koh Y, Park SK, et al. A comparison of the time from sepsis to inception of continuous renal replacement therapy versus RIFLE criteria in patients with septic acute kidney injury. Shock. 2012 Jul;38(1):30-6.

21 McCall CE, Zabalawi M, Liu T, Martin A, Long DL, Buechler NL, et al. Pyruvate dehydrogenase complex stimulation promotes immunometabolic homeostasis and sepsis survival. JCI Insight. 2018 Aug;3(15): 99292. 
22 Yao X, Carlson D, Sun Y, Ma L, Wolf SE, Minei JP, et al. Mitochondrial ROS Induces Cardiac Inflammation via a Pathway through mtDNA Damage in a Pneumonia-Related Sepsis Model. PLoS One. 2015 Oct; 10(10): 0139416

23 Liang D, Huang A, Jin Y, Lin M, Xia X, Chen $\mathrm{X}$, et al. Protective effects of exogenous NaHS against sepsis-induced myocardial mitochondrial injury by enhancing the PGC-1 $\alpha /$ NRF2 pathway and mitochondrial biosynthesis in mice. Am J Transl Res. 2018 May;10(5):142230.

24 Li S. et al. A Novel Mechanism of Mesenchymal Stromal Cell-Mediated Protection against Sepsis: Restricting Inflammasome Activation in Macrophages by Increasing Mitophagy and Decreasing Mitochondrial ROS. Oxid Med Cell Longev. 2018 Feb 13;2018: 3537609.

25 Stone OA, El-Brolosy M, Wilhelm K, Liu X, Romão AM, Grillo E, et al. Loss of pyruvate kinase M2 limits growth and triggers innate immune signaling in endothelial cells. Nat Commun. 2018 Oct;9(1):4077.

26 Banoth B, Cassel SL. Mitochondria in innate immune signaling. Transl Res. 2018 Dec;202: 52-68.

27 Bohr VA. Repair of oxidative DNA damage in nuclear and mitochondrial DNA, and some changes with aging in mammalian cells. Free Radic Biol Med. 2002 May;32(9):804-12.

28 Hill S, Sataranatarajan K, Remmen HV. Role of Signaling Molecules in Mitochondrial Stress Response. Front Genet. 2018 Jul;9:225.

29 Farmer T, Naslavsky N, Caplan S. Tying trafficking to fusion and fission at the mighty mitochondria. Traffic. 2018 Aug;19(8):569-77.

30 Stanzani G, Duchen MR, Singer M. The role of mitochondria in sepsis-induced cardiomy- opathy. Biochim Biophys Acta Mol Basis Dis. 2019 Apr 1;1865(4):759-773

31 Chen JF, Liu H, Ni HF, Lv LL, Zhang MH, Zhang $\mathrm{AH}$, et al. Improved mitochondrial function underlies the protective effect of pirfenidone against tubulointerstitial fibrosis in 5/6 nephrectomized rats. PLoS One. 2013 Dec;8(12):e83593.

32 Nicholls TJ, Gustafsson CM. Separating and Segregating the Human Mitochondrial Genome. Trends Biochem Sci. 2018 Nov;43(11): 869-81.

33 Gangelhoff TA, Mungalachetty PS, Nix JC, Churchill ME. Structural analysis and DNA binding of the HMG domains of the human mitochondrial transcription factor A. Nucleic Acids Res. 2009 Jun;37(10):3153-64.

34 Lyonnais S, Tarrés-Solé A, Rubio-Cosials A, Cuppari A, Brito R, Jaumot J, et al. The human mitochondrial transcription factor $\mathrm{A}$ is a versatile G-quadruplex binding protein. Sci Rep. 2017 Mar;7(1):43992.

35 Chen XJ, Butow RA. The organization and inheritance of the mitochondrial genome. Nat Rev Genet. 2005 Nov;6(11):815-25.

36 Yoshida Y, Izumi H, Torigoe T, Ishiguchi H, Itoh $\mathrm{H}$, Kang D, et al. P53 physically interacts with mitochondrial transcription factor $\mathrm{A}$ and differentially regulates binding to damaged DNA. Cancer Res. 2003 Jul;63(13): 3729-34.

37 Canugovi C, Maynard S, Bayne AC, Sykora P, Tian J, de Souza-Pinto NC, et al. The mitochondrial transcription factor A functions in mitochondrial base excision repair. DNA Repair (Amst). 2010 Oct;9(10):10809.

38 Hayashi Y, Yoshida M, Yamato M, Ide T, Wu Z, Ochi-Shindou M, et al. Reverse of age-dependent memory impairment and mitochon- drial DNA damage in microglia by an overexpression of human mitochondrial transcription factor a in mice. J Neurosci. 2008 Aug; 28(34):8624-34.

39 Suarez J, Hu Y, Makino A, Fricovsky E, Wang $\mathrm{H}$, Dillmann WH. Alterations in mitochondrial function and cytosolic calcium induced by hyperglycemia are restored by mitochondrial transcription factor $\mathrm{A}$ in cardiomyocytes. Am J Physiol Cell Physiol. 2008 Dec; 295(6):C1561-8.

40 Bosmann M, Ward PA. The inflammatory response in sepsis. Trends Immunol. 2013 Mar; 34(3):129-36.

41 Kay E, Scotland RS, Whiteford JR. Toll-like receptors: role in inflammation and therapeutic potential. Biofactors. 2014 May-Jun;40(3): 284-94.

42 Crouser ED, Shao G, Julian MW, Macre JE, Shadel GS, Tridandapani S, et al. Monocyte activation by necrotic cells is promoted by mitochondrial proteins and formyl peptide receptors. Crit Care Med. 2009 Jun;37(6):2000-9.

43 Nakanishi H, Hayashi Y, Wu Z. The role of microglial mtDNA damage in age-dependent prolonged LPS-induced sickness behavior. Neuron Glia Biol. 2011 Feb;7(1):17-23.

44 Hsieh YY, Shen CH, Huang WS, Chin CC, Kuo YH, Hsieh MC, et al. Resistin-induced stromal cell-derived factor-1 expression through Toll-like receptor 4 and activation of p38 MAPK/ NFkB signaling pathway in gastric cancer cells. J Biomed Sci. 2014 Jun;21(1): 59.

45 Yoshino M, Naka A, Sakamoto Y, Shibasaki A, Toh M, Tsukamoto S, et al. Dietary isoflavone daidzein promotes $\mathrm{Tfam}$ expression that increases mitochondrial biogenesis in $\mathrm{C} 2 \mathrm{C} 12$ muscle cells. J Nutr Biochem. 2015 Nov; 26(11):1193-9. 